\title{
Servicios ecosistémicos y equidad social ¿quién controla, quién se beneficia y quién pierde?
}

Améline Vallet ${ }^{1,2}$, Bruno Locatelli,3,4 and Emilia Pramova ${ }^{3}$

\section{Mensajes clave}

- Los actores cumplen diversos roles en la coproducción de servicios ecosistémicos; por ejemplo, pueden ser gestores o beneficiarios directos o indirectos.

- $\quad$ Existen disparidades entre quienes gestionan los servicios ecosistémicos y quienes dependen o se benefician de ellos.

- Las diferentes formas de inequidad asociadas a estos roles pueden poner en riesgo la resiliencia de los sistemas socioecológicos.

- Las asimetrías de poder influyen en los roles de los actores en relación con los servicios ecosistémicos, incluida su participación en la gobernanza de dichos servicios.

- La comprensión de los roles, el poder y las inequidades puede resultar útil para el diseño de mecanismos de gobernanza participativa.

Los ecosistemas de organismos vivos y componentes abióticos producen servicios ecosistémicos por medio de una compleja red de relaciones. Pero también existen "ecosistemas de gestión", que son las redes de actores que participan en la producción y distribución de los beneficios de los servicios ecosistémicos mediante competencia y cooperación. Como en cualquier ecosistema, cada entidad afecta y se ve afectada por las demás en una relación que está en evolución constante.

\section{Los diferentes actores de los servicios ecosistémicos y sus roles: una exploración de la inequidad}

Los ecosistemas saludables, como bosques, pastizales y estuarios, ayudan a regular los caudales de aguas pluviales y el suministro de agua potable, fomentan la polinización de cultivos, estabilizan el suelo y proporcionan madera,

\footnotetext{
1 Ecologie Systématique Evolution, AgroParisTech, CNRS, Université Paris-Sud, Université Paris-Saclay

2 CIRED, AgroParisTech, CIRAD, CNRS, EHESS, Ecole des Ponts

ParisTech, Université Paris-Saclay

3 CIFOR

4 CIRAD, Forests and Societies, University of Montpellier
}

alimentos y plantas medicinales. En conjunto, estos beneficios se conocen como "servicios ecosistémicos" (SE) o "servicios de los ecosistemas".

Es importante tomar en cuenta los SE para la formulación de políticas públicas, dada su considerable contribución al bienestar humano. También es fundamental entender de qué manera funcionan las compensaciones (tradeoffs) entre SE para la planificación y la gestión del paisaje (Mastrángelo et al. 2019). Una situación de compensación se produce cuando un incremento o ganancia en calidad, cantidad o propiedad implica una pérdida en otros aspectos.

En términos más sencillos, en el caso de los SE, esto significa que el incremento de un servicio puede producirse a expensas (de manera directa o indirecta) de otro servicio (Vallet et al. 2018). Por ejemplo, en el Perú, la forestación con especies exóticas como el pino o el eucalipto genera madera e ingresos para los habitantes rurales, pero lleva a una disminución de los recursos hídricos (Bonnesoeur et al. 2019). Por ello, se requiere de una gestión integral del paisaje, que tome en cuenta los diversos SE y sus compensaciones.

En teoría, las rutas hacia el desarrollo sostenible con resultados económicos, ambientales y sociales son atractivas y deseables, pero, en la práctica, estas narrativas de ganar-ganar (win-win) son difíciles de lograr debido a la existencia de compensaciones (Vallet 2018). 
Cuando los responsables de la toma de decisiones, los planificadores y los científicos toman en cuenta las compensaciones, por lo general se centran en sus dimensiones biofísicas, como el espacio físico (por ejemplo, entre comunidades de cuencas hidrográficas aguas arriba y aquellas aguas abajo) o la provisión de diferentes SE (por ejemplo, las compensaciones entre el secuestro de carbono y la regulación hidrológica) (Vallet 2018; Martín-López et al. 2019).

Pero ¿qué sucede con las compensaciones entre el bienestar de diferentes actores relacionados con los SE? Por ejemplo, ¿qué ocurre con las compensaciones entre las personas que gestionan los ecosistemas y aquellas que se benefician de los SE, incluidos los grupos marginados y excluidos, o entre hombres y mujeres? Dichas compensaciones rara vez son exploradas, al igual que los diferentes roles de las personas en relación con la gestión, la regulación y los beneficios de los SE (Mathez-Stiefel et al. 2017).

Las compensaciones entre diferentes SE, y entre el bienestar de diferentes grupos de actores, no solo ocurren debido a los procesos naturales relacionados con la producción de SE. También se producen debido a las intervenciones humanas necesarias para la coproducción de los beneficios que obtenemos de los SE (Lavorel et al. 2020). "Coproducción" significa que, en la mayoría de los casos, la naturaleza no nos proporciona de manera automática estos beneficios. Para obtenerlos, necesitamos gestionar la tecnología, el conocimiento, el capital financiero u otras formas de gestión directa e indirecta de los ecosistemas (Bruley et al. 2020).

En este proceso, ¿quién controla, quién gestiona y quién se beneficia de los SE? Estas son preguntas importantes que se deben explorar. El poder de gestionar e influir en los SE a menudo no es compartido adecuadamente con los grupos que dependen de los ecosistemas o se benefician de ellos. Tales inequidades pueden generar conflictos y poner en riesgo la resiliencia tanto de los ecosistemas como de las comunidades.

\section{Un marco para analizar el papel de los actores}

Desarrollamos un marco analítico sencillo para explorar estas preguntas (figura 1) y lo aplicamos a un estudio de caso en la cuenca del río Mariño, en el Perú. Nuestro marco se basa en la idea de la "cascada de SE" y puede aplicarse a situaciones de la vida real en diferentes contextos. La cascada de SE es similar a una cadena de valor: ilustra el camino que va desde las estructuras y procesos ecológicos, en uno de los extremos, hasta el bienestar de las personas, en el otro (Fedele et al. 2017).

Dentro de la cascada, consideramos dos tipos de interacciones: (1) actividades de gestión que contribuyen al flujo de SE y sus beneficios relacionados; y (2) beneficios recibidos por los. Además, clasificamos las actividades de gestión en indirectas y directas. Las intervenciones humanas directas, como la plantación de árboles, la construcción de infraestructura y la recolección de productos, son solo una de las caras de la gestión de SE, la cual también puede verse influenciada de manera indirecta por los actores que controlan, autorizan o incentivan ciertas acciones. En otras palabras, los gestores directos inciden en el funcionamiento de los ecosistemas y los servicios prestados a la sociedad, mientras que los gestores indirectos facilitan y restringen las actividades de los gestores directos o controlan el uso de los SE (y, por tanto, los beneficios derivados de ellos).

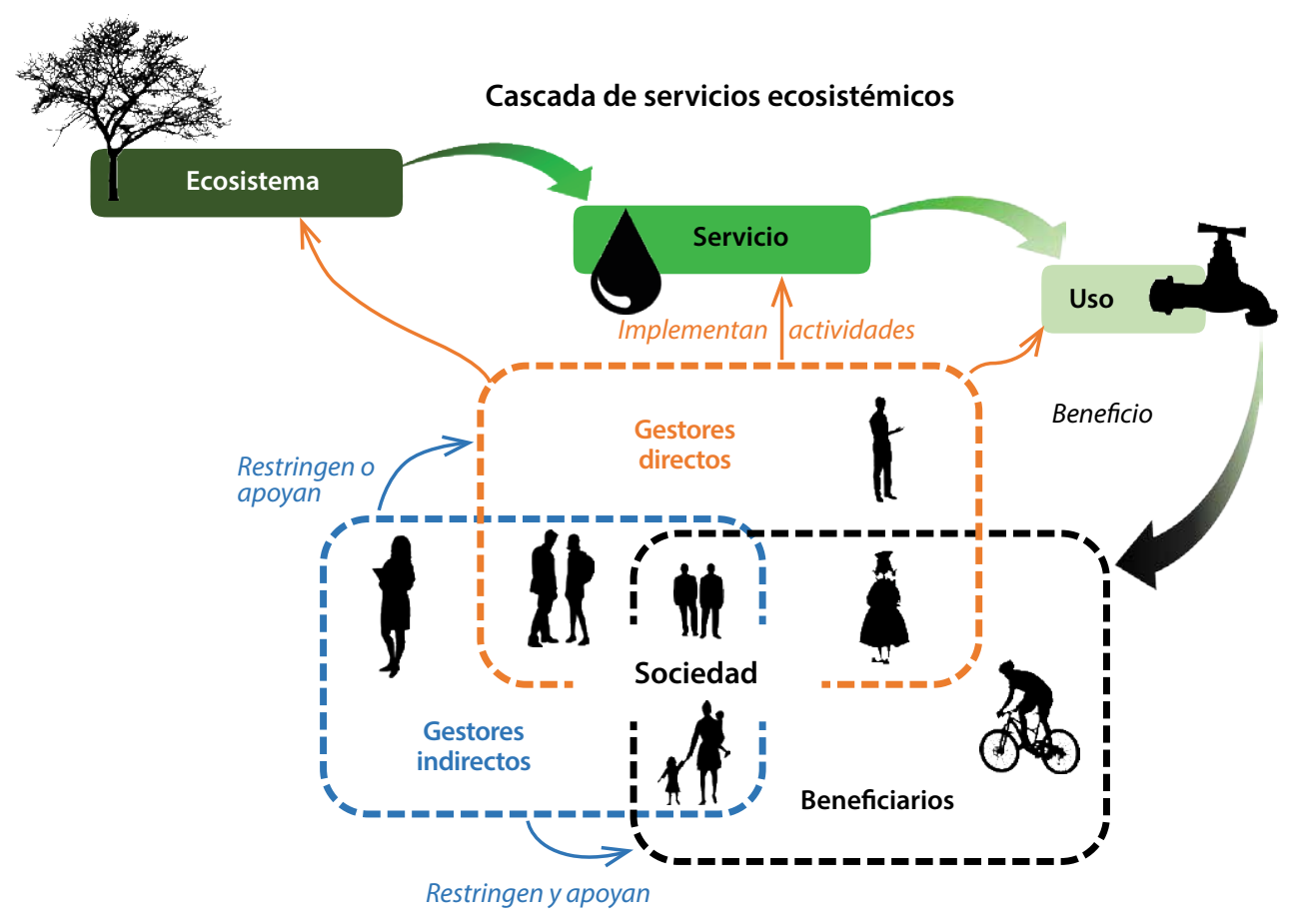

Figura 1. Marco analítico con dos formas de gestión de los servicios ecosistémicos (directa e indirecta), que pueden ocurrir en tres pasos de la cascada de servicios ecosistémicos (ecosistema, servicio y uso). 


\section{Estudio de caso y métodos}

La cuenca del río Mariño (figura 2) se extiende por más de 300 $\mathrm{km}^{2}$ a lo largo de la vertiente oriental de los Andes del sur del Perú, en la región Apurímac, una de las más pobres del país. En ella viven aproximadamente 70000 personas, la mayoría en dos áreas urbanas: Abancay y Tamburco. La agricultura en zonas de gran altitud e intermedias está orientada a la subsistencia, mientras que, en las zonas de baja altitud, tanto la agricultura como la ganadería tienen una orientación comercial y son más intensivas. También hay actividades turísticas en el Santuario Nacional de Ampay, que protege 3635 ha de tierra. Al igual que otros sistemas socioecológicos de montaña, la cuenca del río Mariño proporciona SE importantes pero vulnerables, que contribuyen de manera sustancial al bienestar humano en las tierras altas y bajas (Locatelli et al. 2017).

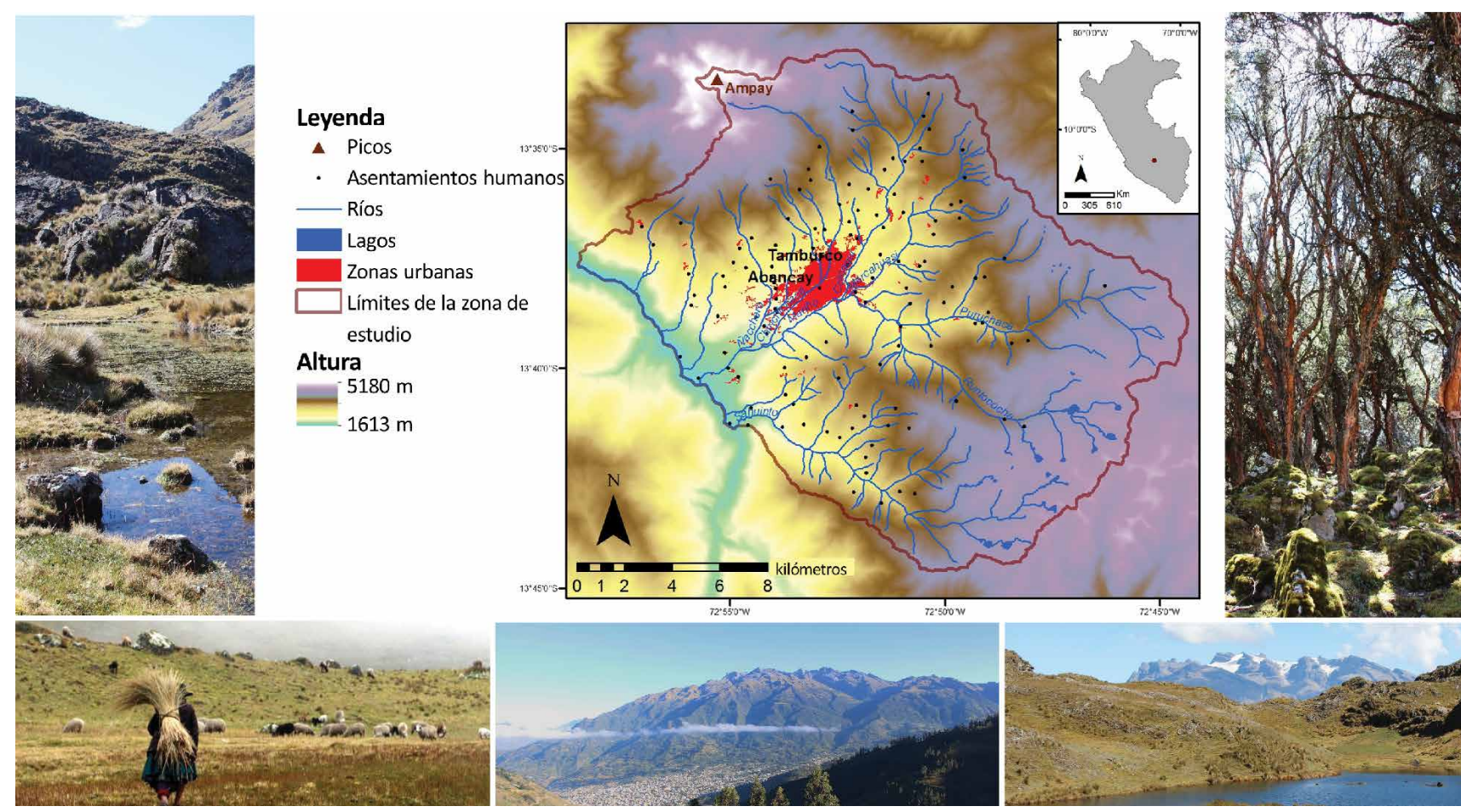

Figura 2. Mapa e imágenes de la zona del estudio de caso.

Fotografías y mapa: Améline Vallet.
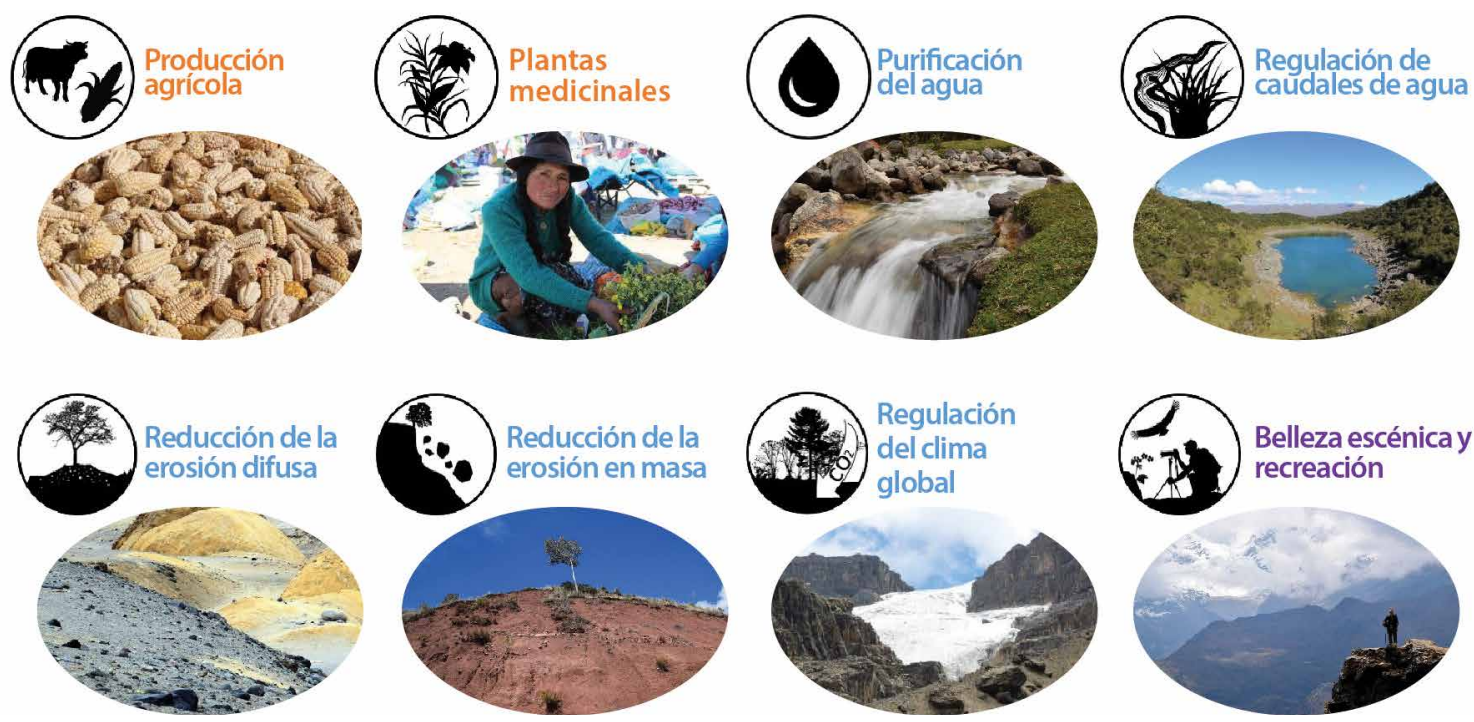

Figura 3. Los ocho servicios ecosistémicos priorizados por los actores locales: dos servicios de aprovisionamiento (texto naranja), cinco servicios de regulación (texto azul) y un servicio cultural (texto violeta).

Fotografías: Améline Vallet y Bruno Locatelli 
En primer lugar, organizamos varios talleres con los actores locales a fin de identificar los SE más importantes y a las instituciones y grupos de actores claves de la zona. Los participantes priorizaron ocho SE importantes (figura 3). Luego, realizamos entrevistas semiestructuradas. Preguntamos a los representantes de 52 instituciones y grupos de interés distintos sobre sus actividades de SE, cómo se benefician de ellas y cómo las gestionan de manera directa o indirecta (en caso las gestionen). Utilizamos esta información para mapear y analizar los diferentes roles de los actores a lo largo de la cascada de SE.

También pedimos a los entrevistados que identificaran con qué otros actores tenían interacción en relación con los ocho SE. Utilizamos esta información para elaborar las redes sociales y lograr una mejor comprensión de las asimetrías de poder. Empleamos herramientas que se aplican comúnmente al análisis de redes sociales (ARS) con el fin de comparar grupos de actores en las redes o explicar la formación de vínculos entre actores. La metodología detallada, la guía de entrevistas semiestructuradas y los resultados cualitativos para los ocho SE se encuentran disponibles en los artículos de revistas académicas de acceso abierto relacionados (Vallet et al. 2019, 2020).

\section{Disparidades entre quienes se benefician y quienes gestionan}

Descubrimos que los grupos que dependían o se beneficiaban de los SE no estaban necesariamente involucrados en su gestión. Por ejemplo, los beneficiarios de la regulación global del clima eran las poblaciones rurales y urbanas, las empresas locales (piscifactorías, turismo, agroindustrias), así como varias instituciones públicas. Sin embargo, quienes tenían una participación activa en la mitigación del cambio climático eran principalmente ONG e instituciones ambientales del sector público (mediante programas de forestación, reforestación y reducción de emisiones).

Los gestores directos eran en su mayoría actores locales, empresas y la sociedad civil, mientras que los gestores indirectos eran a menudo organizaciones de alcance nacional y otros grupos del sector público (que facilitaban o restringían la gestión y el uso directos). Por ejemplo, los gestores directos de las plantas medicinales eran los habitantes rurales que las cosechaban, transportaban y vendían en los mercados de la ciudad o que cultivaban especies de estas plantas en sus campos. Por otro lado, los gestores indirectos eran las instituciones públicas encargadas de controlar y autorizar las actividades relacionadas con la flora silvestre o con áreas protegidas. En la mayor parte de los SE, los grupos que participaban en su gestión directa tenían muchas probabilidades de ser también beneficiarios. Por ejemplo, los agricultores que gestionaban directamente la producción agrícola y, por tanto, coproducían estos SE se beneficiaban de los productos agrícolas mediante el comercio y la generación de ingresos.

Los actores involucrados en el primer paso de la cascada, es decir, el nivel del ecosistema, tenían el mayor control sobre los beneficios recibidos en el último paso, es decir, el nivel de los beneficios. Por ejemplo, los agricultores encargados de la gestión de la producción agrícola mediante la siembra y el cultivo (niveles del ecosistema y del servicio) tenían influencia sobre la cantidad de alimentos disponibles para los consumidores. Pero esta influencia solo estaba relacionada con la cantidad o calidad de los SE, y no con los aspectos de distribución (es decir, quién podía recibir estos beneficios). De manera similar, los agricultores y las comunidades tenían poder para influir en la gestión de la tierra y el suministro de agua (por ejemplo, mediante la reforestación y la protección de los humedales), pero carecían de poder para decidir sobre la asignación de uso de agua entre varios usuarios. En cambio, la Autoridad Nacional del Agua (ANA) carecía de poder en términos de la gestión territorial, pero sí contaba con él en lo referente al control y autorización del consumo de agua.

Los SE hidrológicos en particular estaban vinculados a tales problemas de equidad y justicia. En la cuenca del río Mariño, el agua es canalizada principalmente río abajo, hacia las áreas urbanas, por EMUSAP (Empresa Municipal de Servicios de Agua Potable y Alcantarillado), el proveedor local de servicios de agua potable autorizado por la ANA. En el río aguas abajo de la represa Rontococcha (en la parte oriental de la cuenca), la extracción de agua para su uso en la ciudad traía como resultado una escasez de agua para otros usos, por ejemplo, la agricultura. Esto llevó a conflictos entre las comunidades agrícolas de las tierras altas y aquellas de las tierras bajas en torno al acceso al agua. Los agricultores que se encontraban río abajo también estaban preocupados por problemas relacionados con la calidad del agua, ya que la poca que llegaba hasta sus campos estaba contaminada por la ciudad.

Estos conocimientos pueden servir de base para debatir sobre una gestión más equitativa de los SE en un área en particular. Por ejemplo, pueden utilizarse en las discusiones relacionadas con el esquema de pagos propuesto para los SE hidrológicos en la cuenca. Dicho esquema tiene el potencial de brindar oportunidades para la acción colectiva, la participación directa y la coordinación entre los diversos actores.

Los diversos roles que desempeñan los actores en la cascada de SE, y las inequidades y asimetrías de poder asociadas a ellos, pueden explicarse por sus diferencias en derechos y prerrogativas, acceso a recursos y otros tipos de capital, y limitaciones relacionadas con su ubicación, las instituciones y las normas sociales. Las asimetrías de poder también pueden explicarse por las relaciones formales o informales entre actores.

\section{Análisis de redes sociales para destacar las asimetrías de poder y los conflictos relacionados}

Realizamos un análisis de redes sociales (ARS) con el fin de conocer mejor las relaciones de poder entre gestores y beneficiarios de los SE. Los principales objetivos del ARS son mapear y medir las 
relaciones (vínculos relacionales) que conectan a individuos u organizaciones (actores) en una red, e identificar actores o grupos clave y aislados (Di Gregorio et al. 2019). Este análisis examina y explica el poder en función de las posiciones de los actores en una red determinada. Por ejemplo, los actores que tienen una posición central en las redes de comunicación o intercambio de conocimientos (es decir, con mediciones de centralidad altas) se encuentran mejor posicionados para influir en los demás. Existen diferentes mediciones de la centralidad que se pueden utilizar para ilustrar este poder. Aquí nos enfocamos en la centralidad de grado, que muestra el número de vínculos relacionales que mantiene un actor y nos indica cuántas conexiones directas de "un grado" tiene este actor con otros actores en la red.

A partir de estudios previos realizados por sociólogos, analizamos dos formas de poder relacional dentro de nuestro ARS: dominación e influencia. La dominación es la capacidad de ofrecer o negar beneficios o efectos nocivos por medio de la fuerza, coerción o autoridad. La influencia consiste en proporcionar información para cambiar la opinión, actitud o comportamiento de un actor. Las asimetrías de poder corresponden a la distribución desigual de estas dos dimensiones de poder entre los actores (tabla 1).

Además de identificar las asimetrías de poder, también apuntamos a comprender los diferentes tipos de poder que manejan los actores. Utilizamos un marco propuesto por Knoke (1990), que distingue entre cuatro tipos de actores en función de sus niveles de influencia y dominación (figura 4): actores con poder coercitivo, autoritario, persuasivo e igualitario (es decir, débil). También nos interesaba conocer de manera específica quién ejercía poder sobre quién en relación con la gobernanza de los SE y cuáles eran las consecuencias de ello en términos de conflictos.

Tabla 1. Los dos tipos de asimetrías de poder consideradas en el estudio y su impacto en la gobernanza de los SE.

\begin{tabular}{ll}
\hline & Asimetría dentro de una forma de pode \\
\hline $\begin{array}{l}\text { Representación } \\
\text { gráfica }\end{array}$ & Algunoncia actores son más influyentes \\
& que otros (en este caso, A) \\
\hline Definición & $\begin{array}{l}\text { Intercambio eficiente de infor- } \\
\text { mación (si A está cumpliendo con } \\
\text { su trabajo) (Bodin 2017; Guerrero } \\
\text { et al. 2020) } \\
\text { positivos en la } \\
\text { gobernanza de } \\
\text { los SE }\end{array}$ \\
$\begin{array}{l}\text { Contribución a la sostenibilidad } \\
\text { (si A comparte información que } \\
\text { ayuda a las acciones de sostenibi- } \\
\text { lidad) (Fischer 2014) }\end{array}$
\end{tabular}

Asimetría entre diferentes formas Dominación de poder

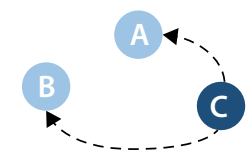

Algunos actores son más dominantes que otros (en este caso, C)

- Coordinación eficiente (si C está cumpliendo con su trabajo) (Bodin 2017; Guerrero et al. 2020)

- Contribución a la sostenibilidad (si $C$ impulsa acciones de sostenibilidad) (Fischer 2014)

Los actores más influyentes $(\mathrm{A})$ no son los más dominantes (C) y viceversa

- Riesgo limitado de abuso por parte de un actor autoritario que concentra influencia y poderes de dominación, por la existencia de un poder compensatorio (por ejemplo, $C$ puede denunciar los abusos de $\mathrm{A}$, actuando como denunciante) (Fung et al. 2003; Crona y Bodin 2010)

- Eficiencia por la distribución de responsabilidades y especialización (por ejemplo, A se especializa en compartir información)

- Diversos catalizadores del cambio (A puede estimular el cambio y desafiar la inercia de $C$, o viceversa) (Crona y Bodin 2010)

Impactos
negativos en la
gobernanza de
los SE

- Concentración de poder sin poder compensatorio, que puede conducir a manipulación (el actor poderoso A puede filtrar o distorsionar la información) o bloqueo (el actor poderoso A puede bloquear o restringir el intercambio de información) (Crona y Bodin 2010; Barnes et al., 2016)

- Falta de diversidad en la información (lo que puede limitar el aprendizaje colectivo)
- Concentración de poder sin poder compensatorio, que puede conducir a inercia (el actor poderoso $\mathrm{C}$ puede restringir voluntariamente las acciones o ser ineficiente) o abuso de autoridad (C puede actuar en su propio beneficio) (Bodin 2017; Guerrero et al. 2020)

- Falta de diversidad en el liderazgo (lo que puede limitar la innovación)
- Riesgo de poder centralizado encubierto si $\mathrm{A}$ y $\mathrm{C}$ son aliados (por ejemplo, si $C$ es un gobierno autoritario y $\mathrm{A}$ es su agencia de prensa)

- Riesgo de bloqueo o conflictos por disputas de poder si A y C no están de acuerdo (y A hace campaña en contra de $\mathrm{C}$ o $\mathrm{C}$ obliga a A a guardar silencio) 


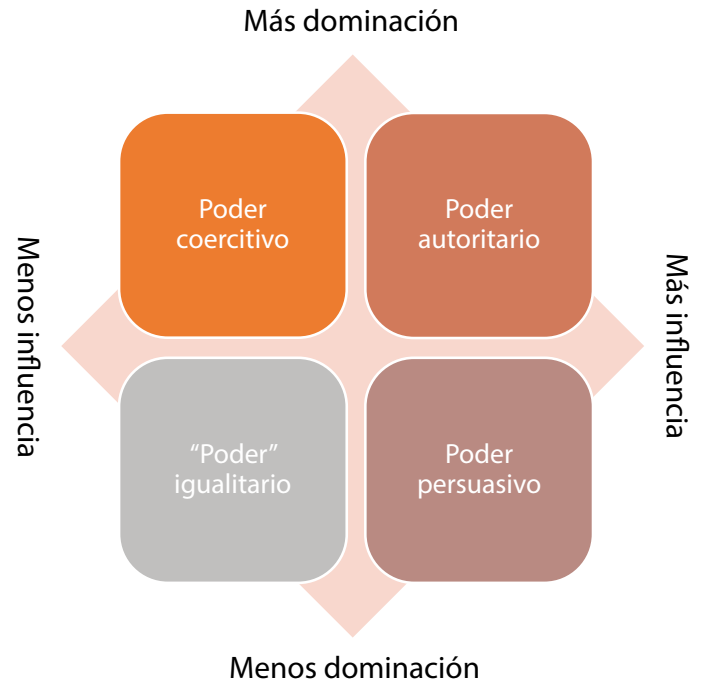

Figura 4. Los actores presentan cuatro tipos de poder según la magnitud de su influencia y dominación (según Knoke 1990).

Para desarrollar el ARS, preguntamos a nuestros entrevistados con quiénes interactuaban, de acuerdo con ocho tipos de relaciones que representan influencia, dominación y cooperación (figura 5). Al final del proceso de entrevista, también les pedimos que indicaran con qué actores habían tenido relaciones tensas o problemáticas y que explicaran el porqué.

Combinamos las relaciones de influencia para formar una red de influencia $(1+2)$ y procedimos de manera similar con las redes de dominación (3+4) y cooperación $(5+6+7+8)$ (figura 6). Consideramos que la dominación es una red direccional (es decir, un actor se identifica como el iniciador de la relación y el otro como el receptor) y que las redes de influencia y cooperación son no direccionales (es decir, ambos actores participan como iguales en la relación).

Al examinar el poder, encontramos que las dos redes de influencia y dominación estaban poco correlacionadas, lo que sugiere que los actores influyentes no son necesariamente dominantes. Ambas redes también diferían en términos de su estructura centro/periferia (figura 7). En general, el centro denso y cohesivo de una red abarca a los actores principales que tienen una cantidad enorme de vínculos relacionales, mientras que los actores de la periferia poco conectada mantienen pocos vínculos relacionales.

Las estructuras centro-periferia corresponden a la distribución de poder entre los diferentes tipos de actores. Los actores del sector de negocios mostraron puntuaciones de centralidad significativamente más bajas en la red de influencia, mientras que los actores del sector público mostraron puntuaciones de centralidad significativamente más altas en la red de dominación.

El tipo de poder que manejaban los actores también dependía de su rol. Los beneficiarios de los SE tenían más probabilidades de encontrarse en el grupo de actores débiles. Por el contrario, los gestores directos tenían más probabilidades de contar con

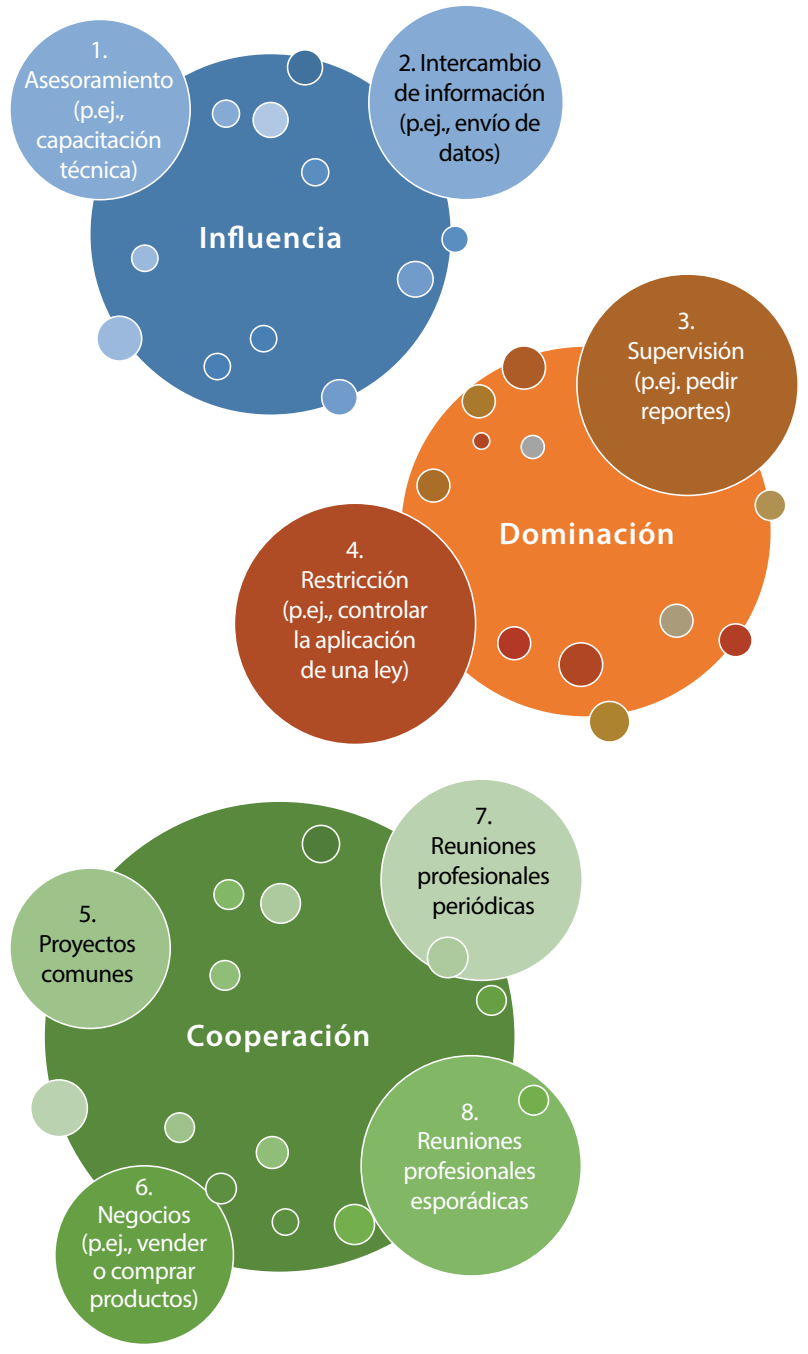

Figura 5. Los ocho tipos de relaciones (texto en círculos pequeños) que representan influencia, dominación y cooperación (los diámetros de los círculos no son representativos).

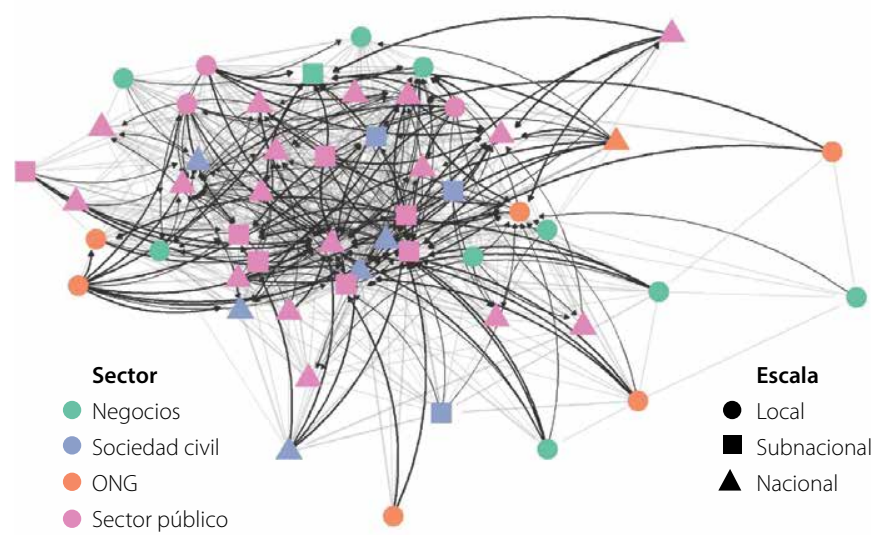

Figura 6. Redes de influencia (vínculos rectos color gris claro) y de dominación (vínculos curvos color gris oscuro). El grosor de los vínculos es proporcional al peso de la relación (entre 0 y 1 ). 
poder persuasivo y los gestores indirectos, poder autoritario o coercitivo. De manera similar, los actores del sector público tenían más probabilidades de formar relaciones de influencia, en tanto que ocurría lo contrario en el caso de los actores de negocios. Los actores de negocios y de la sociedad civil tenían más probabilidades de ser los receptores de una relación de dominación, en la mayoría de los casos iniciada por actores del sector público.

La existencia de una relación de cooperación también afectó positivamente la formación de vínculos de influencia y dominación, es decir, dos actores que cooperaban tenían más probabilidades de influirse o dominarse entre sí. Los actores más centrales de la red de cooperación tenían más probabilidades de ser los iniciadores de las relaciones de dominación.

Nuestros análisis estadísticos como parte del ARS mostraron que la existencia de una relación de dominación entre dos actores aumentaba en gran medida la probabilidad de ocurrencia de un conflicto. Y grandes diferencias en los puntajes de centralidad de la dominación entre dos actores incrementaban aún más esta probabilidad. Esto quiere que había mayor probabilidad de que surgieran conflictos cuando estaban involucrados actores realmente poderosos junto con actores realmente débiles. En cambio, había una menor probabilidad de conflictos entre actores de un mismo sector.

Red de influencia

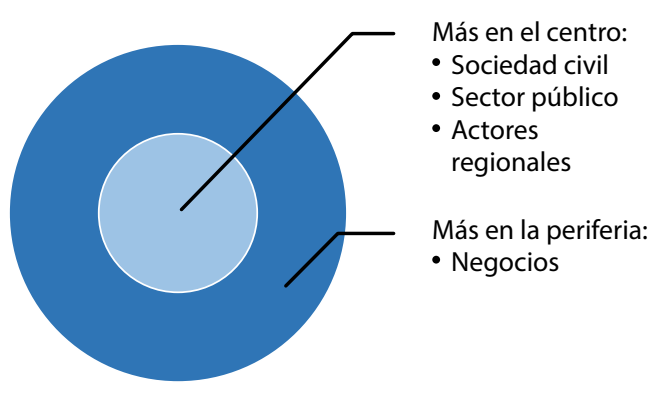

Red de dominación

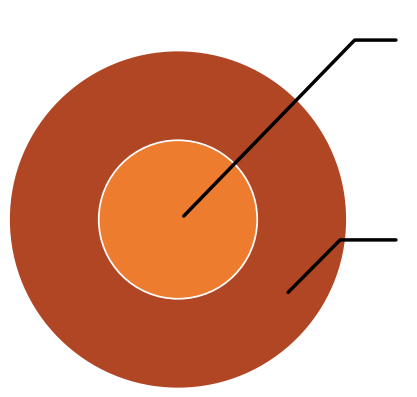

Más en el centro :

- Sector público

Más en la periferia :

- ONG

- Otros actores

nacionales o

internacionales

Figura 7. Tipos de actores que se encuentran con mayor frecuencia en el centro y en la periferia de las redes de influencia y dominación.

\section{Observaciones finales: poder, inequidad y gobernanza de los ecosistemas}

Nuestros resultados demuestran que las asimetrías de poder están asociadas con los conflictos, que a su vez pueden influir en la resiliencia de los sistemas socioecológicos, en nuestro caso la resiliencia de la cuenca del río Mariño. Las asimetrías de poder también pueden generar desconfianza entre las instituciones responsables de diferentes aspectos de la gobernanza de los SE.

El poder y las inequidades relacionadas con él pueden presentar diversos aspectos. Pueden surgir debido a los diferentes roles de los actores en relación con los SE; los diferentes derechos, recursos y ubicaciones de los actores; y las relaciones que estos establecen. Estos aspectos se encuentran también interrelacionados; influyen unos sobre otros, y es posible explorarlos utilizando nuestro marco de cascada de SE, el ARS y otros métodos.

El marco de cascada de SE destaca los diferentes roles de los actores en la gestión de los ecosistemas y los SE, y en el uso de sus beneficios. También puede ilustrar las desigualdades vinculadas a estos roles. Las asimetrías de poder se pueden explorar más a fondo con el ARS.

Comprender las redes sociales de gobernanza de los SE puede ayudarnos a entender tanto las condiciones propicias como los obstáculos para una gestión sostenible y equitativa de los ecosistemas. La identificación de actores clave, actores aislados y aquellos que pueden promover nuevas colaboraciones o relaciones entre grupos puede ser útil para la planificación del uso de la tierra, la resolución de conflictos y el diseño de mecanismos de gobernanza participativa.

\section{Agradecimientos}

Los socios financiadores que han apoyado esta investigación incluyen el programa de investigación e innovación H2020 de la Unión Europea (proyecto SINCERE), la Iniciativa Climática Internacional (IKI) del Ministerio Federal de Medio Ambiente, Conservación de la Naturaleza, Construcción y Seguridad Nuclear de Alemania (BMUB), la Agencia Noruega para la Cooperación al Desarrollo, el Ministerio de Transición Ecológica e Inclusiva de Francia y el Programa de Investigación del CGIAR sobre Bosques, Árboles y Agroforestería (CRP-FTA) con apoyo financiero del Fondo CGIAR. Los autores agradecen a Helvetas, Bosques Andinos, CEDES e IDMA por su apoyo logístico y ayuda en la organización de los talleres y entrevistas; así como a todos los entrevistados por su tiempo e información útil.

\section{Referencias}

Bruley E., Locatelli B., Lavorel S., 2020. Nature's contributions to people: Co-producing quality of life from multifunctional landscapes. Ecology and Society. 
Bonnesoeur V., Locatelli B., et al., 2019. Impacts of forests and forestation on hydrological services in the Andes: A systematic review. Forest Ecology and Management 433: 569-584. http://doi. org/10.1016/j.foreco.2018.11.033

Di Gregorio M., Fatorelli L., et al., 2019. Multi-level governance and power in climate change policy networks. Global Environmental Change 54: 64-77. http://doi.org/10.1016/j.gloenvcha.2018.10.003

Fedele G., Locatelli B., Djoudi H., 2017. Mechanisms mediating the contribution of ecosystem services to human well-being and resilience. Ecosystem Services 28A: 43-54. http://doi.org/10.1016/j. ecoser.2017.09.011

Knoke D., 1990. Political networks: the structural perspective. Cambridge University Press.

Lavorel S., Locatelli B., et al., 2020. Co-producing ecosystem services for adapting to climate change. Philosophical Transactions of the Royal Society B 375: 20190119. http://doi.org/10.1098/ rstb.2019.0119

Locatelli B., Lavorel S., et al.., 2017. Characteristic trajectories of ecosystem services in mountains. Frontiers in Ecology and the Environment 15(3): 150-159. http://doi.org/10.1002/fee.1470

Martín-López B., Felipe-Lucia M.R., et al., 2019. A novel telecoupling framework to assess social relations across spatial scales for ecosystem services research. Journal of Environmental Management 241: 251-263. http://doi.org/10.1016/j.jenvman.2019.04.029

Mastrángelo M.E., Perez-Harguindeguy N. et al., 2019. Key knowledge gaps to achieve global sustainability goals. Nature Sustainability 2: 1115-1121. http://doi.org/10.1038/s41893-019-0412-1

Mathez-Stiefel S.L., Peralvo M., et al., 2017. Research priorities for the conservation and sustainable governance of Andean forest landscapes. Mountain Research and Development 37(3): 323-339. http://doi.org/10.1659/MRD-JOURNAL-D-16-00093.1

Vallet A., 2018. Tradeoffs between ecosystem services: From landscapes to stakeholders. PhD Thesis, Paris-Saclay University, AgroParisTech, Paris, France.

Vallet A., Locatelli B., et al., 2018. Relationships between ecosystem services: Comparing methods for assessing tradeoffs and synergies. Ecological Economics 150: 96-106. http://doi.org/10.1016/j. ecolecon.2018.04.002

Vallet A., Locatelli B., et al., 2019. Linking equity, power and stakeholders' roles in relation to ecosystem services. Ecology and Society 24 (2):14. http://doi.org/10.5751/ES-10904-240214

Vallet A., Locatelli B., et al., 2020. Power asymmetries in social networks of ecosystem services governance. Environmental Science and Policy 114: 329-340. http://doi.org/10.1016/j.envsci.2020.08.020

\begin{tabular}{|l|l|l|l|l}
\hline & $\begin{array}{l}\text { Esta investigación fue realizada por CIFOR como parte del Programa de Investigación de CGIAR sobre } \\
\text { Bosques, Árboles y Agroforestería (FTA). El FTA es el programa de investigación para el desarrollo } \\
\text { más grande del mundo, dedicado a mejorar el papel de bosques, árboles y la agroforestería para el } \\
\text { desarrollo sostenible, la seguridad alimentaria, y frente al cambio climático. CIFOR dirige el programa }\end{array}$ \\
FTA en asociación con Bioversity International, CATIE, CIRAD, ICRAF, INBAR y TBI. \\
La investigación del Programa FTA cuenta con el apoyo del Fondo Fiduciario del CGIAR: cgiar.org/ \\
funders/
\end{tabular}
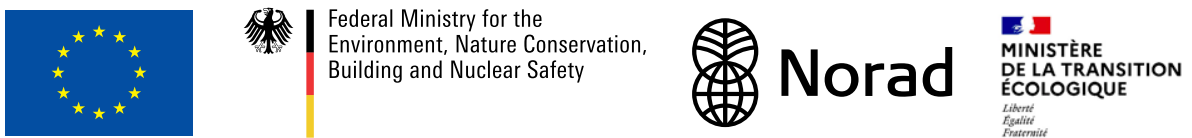
desarrollando las capacidades de sus socios y dialogando activamente con todos los actores involucrados, para informar sobre las políticas y las prácticas que afectan a los bosques y a las personas. CIFOR es un centro de investigación CGIAR y lidera su Programa de Investigación sobre Bosques, Árboles y Agroforestería (FTA por sus siglas en inglés). Nuestra sede central se encuentra en Bogor, Indonesia, y contamos con oficinas en Nairobi, Kenia; Yaundé, Camerún; Lima, Perú, y Bonn, Alemania.

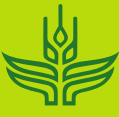

CGIAR 